\title{
Comunicación

\section{Buscando la sostenibilidad del periodismo de investigación sin ánimo de lucro}

Seeking the sustainability of investigative, non-profit, journalism

\author{
JOSÉ LUIS REQUEJO ALEMÁN1
}

Diez años después de la reactivación del periodismo de investigación sin ánimo de lucro en Estados Unidos, distintas voces le reclaman una mayor sostenibilidad. Hasta ahora, sus ingresos principales han provenido de grandes fundaciones. Sin embargo, como recuerda Edward Wasserman (2011) "Ir periódicamente a la gente rica para pedirles dinero no es un modelo de negocio real". Tomando como base el prestigioso modelo Canvas, creado en 2004, este trabajo valora la sostenibilidad de este tipo de periodismo.

PALABRAS CLAVE: Emprendimiento en periodismo, periodismo de investigación sin ánimo de lucro, periodismo de investigación, modelo de negocio, modelo Canvas.
Ten years after the revival of non-profit investigative journalism in the United States, a variety of voices demand more sustainability. So far, such journalistic practice's main source of income came from large foundations. But, as Edward Wasserman (2011) recalled, attending "regularly to rich people asking for money is not a real business model". Based on the model developed by Canvas, established in 2004, this paper evaluates the sustainability prospects for this kind of journalism.

KEY WORDS: Entrepreneurial journalism, investigative journalism, non-profit journalism, media business model, Canvas business model.

1 Universidad Carlos III de Madrid, España.

Correo electrónico: jlrequej@hum.uc3m.es

Madrid 133 (Edificio Ortega y Gasset), 28903; Getafe, Madrid, España. 


\section{INTRODUCCIÓN}

En su modelo tradicional, el periodismo se financiaba por tres vías: suscripciones y honorarios de quiosco (Osterwalder \& Pigneur, 2010); elevadas tarifas publicitarias a cambio de un acceso privilegiado a grandes públicos (Greer, 2004), y avisos, anuncios o publicidad clasificada. Sin embargo, la irrupción de las nuevas tecnologías en el periodismo y la apatía de su entorno empresarial 2 ocasionaron que este tipo de financiamiento decayera hasta amenazar su existencia (Campos, 2010) y saldarse con la muerte de varios medios de comunicación (Armstrong, 2008).

La industria de diarios está librando la batalla de modo desigual. Anclada a sus gigantescas rotativas, intenta salvar sus imprentas y su tecnología, junto a su misión periodística. Ambos objetivos son disociables en una época en la que el soporte ha dejado de ser relevante (Osterwalder \& Pigneur, 2010). Este empeño por sobrevivir les ha pasado factura a los medios tradicionales, restando recursos a la investigación periodística. Un informe realizado por Pew Research Center para el Project for Excellence in Journalism, certifica que, desde el año 2000, los diarios estadounidenses han perdido mil 600 millones de dólares anuales en capacidad para cubrir y editar noticias. En los tres últimos años la industria de diarios ha cortado miles de trabajos de reporteros y editores a tiempo completo (Stverak, 2010).

Lejos de mirar este panorama con pesimismo, algunos profesionales se están esforzando por reconvertirse laboralmente, 3 emprendiendo nuevas y pequeñas empresas periodísticas, ${ }^{4}$ con el objeto de seguir cumpliendo un importante rol para la sociedad. Algunas de estas inicia-

2 Al respecto, todavía se recuerda la conocida frase del presidente del directorio del Washington Post, Donald Graham, en el año 2001: "Ingresos significa aquí publicidad. Y no va a significar nada más en el futuro" (Greer, 2004, p. 107).

3 Brand Houston (2009), de la Universidad de Illinois, señala que el aumento de este tipo de instituciones sin fines de lucro tiene que ver también con los recientes despidos masivos en la industria periodística.

4 Algunos de los casos más llamativos son los de David Cohn (2009) y, más recientemente el de Burt Herman, de Hacks/Hackers (Hernández, 2010). 
tivas se especializan solo en periodismo de investigación y reciben el nombre de Nonprofit Investigative Journalism o periodismo de investigación sin ánimo de lucro. Actualmente, suman más de 700 dentro de Estados Unidos (Lozano, 2010).

Nuestra principal misión es desarrollar el periodismo de investigación más como una fuerza moral, de ahí que nuestra tarea principal consista en crear historias de alto impacto en la arena pública. Sin embargo, esto requiere recursos para proporcionarle un futuro a este trabajo, asegura Richard J. Tofel, gerente general de ProPublica 5 uno de los premiados en 2009 con un fondo para buscar y consolidar modelos de negocio para el periodismo de investigación, otorgado por la Fundación Knight (Fest, 2009, traducción propia).

La denominación "sin ánimo de lucro" de este tipo de iniciativas puede ser confusa en un primer momento. A primera vista, parece como si este tipo de periodismo estuviera liberado de las presiones de los flujos de capital y de la rentabilidad, que oprimen al mundo de los negocios. Sin embargo, esto no es así (Stverak, 2010). Una organización sin ánimo de lucro está al servicio del bien público por estar libre de deudas y ganancias, pero eso no significa que pueda plantear su vida a costa de pedir colaboraciones: "Ir a la gente rica periódicamente pidiendo dinero no es un modelo de negocio real" (Wasserman, 2011).

La "autosostenibilidad" o simplemente "sostenibilidad" es la nueva palabra clave en este asunto (Alves, comunicación personal, 6 de mayo; McLellan, 2010a; Walton, 2010). Cuando el New England Center for Investigative Reporting fue creado en enero de 2009, la Fundación

5 Una prueba de esta afirmación es el Premio Pulitzer de Periodismo de Investigación 2010, otorgado a la organización de periodismo de investigación sin ánimo de lucro, ProPublica, el 12 de abril de ese año, por un reportaje realizado por la periodista Sheri Fink, sobre una historia referida a un hospital levantado inmediatamente después del huracán Katrina (ella compartió el premio con Bárbara Laker y Wendy Ruderman del Philadelphia Daily News). El coste aproximado de la historia: 400 mil dólares. Lo más llamativo del financiamiento: compartido entre ProPublica, la fundación Kaiser, The New York Times y la propia Fink (Benton, 2010). 
Knight puso 250 mil dólares para financiar la incipiente empresa. En 2012, esta misma fundación aportó otros 400 mil dólares, con la condición de que se encuentre un modelo de autosostenibilidad.

Precisamente, para analizar la sostenibilidad del modelo de negocio del periodismo sin ánimo de lucro, este trabajo usará como patrón de análisis el modelo Canvas, creado en 2004, probado en todo el mundo y utilizado por organizaciones como IBM, Ericsson, Deloitte, el Gobierno de Canadá, y muchos otros (Osterwalder \& Pigneur, 2010). Opto por este modelo a sugerencia del profesor Alves (2011) y debido a que su entendimiento casi intuitivo facilita la descripción del negocio y propicia la discusión.

\section{EL MODELO CANVAS}

Para Canvas un modelo de negocio es "la descripción racional de cómo las organizaciones crean, capturan y distribuyen valor" (Osterwalder \& Pigneur, 2010, p. 14).

Como se recordará, el modelo Canvas tiene cuatro grandes partes básicas, divididas a su vez en nueve bloques específicos (Osterwalder \& Pigneur, 2010):

1. Infraestructura. Primero la empresa debe identificar cuáles son sus actividades clave. Esto no solo abarca las actividades de subsistencia, sino también las que le permiten generar un producto único en su género de tal forma que la empresa y sus trabajadores sean capaces de visualizar aquellas acciones esenciales y distintivas. A partir de ahí, los emprendedores deben identificar cuáles son sus recursos imprescindibles y acordar cuáles son o deberían ser sus socios estratégicos.

2. Oferta. También llamada "propuesta de valor". Es el conjunto de servicios, productos y oferta de negocio encaminados a satisfacer una demanda real del consumidor. Esto es lo que debe diferenciar a la empresa de sus competidores, tanto en cantidad (relación calidad/ precio), como en calidad (una experiencia de usuario única).

3. Clientes. Para conseguir un diseño efectivo, un modelo de negocio debe identificar con claridad a qué público quiere atender para conocer en detalle sus necesidades, la manera de aproximarse e 
introducirse en su vida y para establecer el modo más efectivo de cobro. En esta misma línea, es importante la elección cuidadosa de los canales de distribución; es decir, las diferentes vías a través de las que se va a hacer llegar las propuestas de valor. Por lo general, deben ser rápidas y eficientes, y pueden ser propias o de terceros, o una combinación de ambas. Pero el cometido no termina allí. Para asegurar el éxito de un negocio, las distintas empresas deben tener muy claro la relación que tienen que establecer con el cliente. El modelo Canvas formula hasta cinco opciones posibles: a) con asistencia personal antes, durante y después del consumo del producto o servicio. Se trata de una relación permanente con el usuario final, pero que somete a la empresa y a su personal a un desgaste continuo; b) autoservicio, donde es el propio cliente quien se sirve y prepara sus propios productos o servicios, en una combinación que se ajusta a su medida; c) servicios automáticos, donde una máquina o robot asiste al cliente para mejorar su experiencia como usuario o consumidor; d) comunitarias, donde la comunidad crea un escenario de aprendizaje para compartir y resolver problemas, y finalmente, e) una relación de cocreación, en la que el usuario final introduce un input indispensable para dar por terminado el producto o servicio que se brinda y pasa a formar parte de la cadena de montaje.

4. Financiamiento. El primer factor que está incluido en este apartado es lo que se denomina estructura de costes. Este factor tiene dos ámbitos que deben mantenerse en equilibrio. Uno es el manejo de costes, cuyo énfasis está en dimensionar la economía de la empresa para que se consiga la máxima eficiencia con el menor gasto posible, evitando derroches innecesarios. Además, esta fase debe guardar un equilibrio con el "manejo del valor", en donde se debe invertir, con prudencia, pero sabiendo que, de lo contrario, la empresa perdería su norte y el impulso vital. A la estructura de costes se suma el flujo de ingresos: la manera cómo la compañía obtiene ingresos de cada segmento de clientes. Canvas contempla hasta siete opciones posibles: a) la venta de activos o la venta de los derechos de propiedad sobre un activo; b) cuota de uso, a cambio del uso temporal de un servicio; c) tasas de suscripción, a cambio del uso continuo de un bien o servicio; d) renta, o cobro por el uso de un bien durante 
un determinado periodo de tiempo; e) licencia, o cobro por el uso de algo con propiedad intelectual; f) honorarios por servicios, generalmente entre dos personas a cambio de comercializar un producto; g) publicidad, donde podría también caber el patrocinio.

No se aplicará este modelo a una iniciativa de periodismo sin ánimo de lucro en particular, sino que se usará el esquema para organizar los datos de distintas empresas recopiladas en una revisión bibliográfica previa, sumando además la opinión de algunos expertos. Lo que se recoge a continuación es un ejercicio de análisis crítico realizado en marzo de 2012.

\section{APLICACIÓN DEL MODELO CANVAS AL PERIODISMO DE INVESTIGACIÓN SIN ÁNIMO DE LUCRO}

\section{Infraestructura}

Una de las principales actividades clave que exige una nonprofit es garantizar la exoneración de impuestos para la propia empresa y para los que colaboren con ella. Bajo la figura legal 501(C) (3) de Estados Unidos, este tipo de empresas y sus colaboradores quedan exentos de impuestos y pueden postular a financiamientos adicionales para cubrir los costes de sus proyectos, sin afectar a sus operaciones ordinarias. Este ahorro por lo general se invierte en tecnología y/o en talento periodístico, buenos fichajes. Sin embargo, no se trata de un modelo consolidado, sino de un punto de partida para que, quien esté interesado, siga indagando hasta encontrar su propia fórmula. De acuerdo con lo investigado hasta el momento, los modelos que han tenido mayor éxito empresarial en el corto plazo, es decir, que se han conseguido financiar con holgura, son los modelos mixtos, que consiguen mezclar la condición legal sin ánimo de lucro con su contrario y en una misma compañía. Este es el caso de ProPublica y de The Huffinton Post. Estos modelos mixtos, que en su momento supusieron un auténtico quebradero de cabeza legal para sus diseñadores, podrían ser objeto de un análisis más detallado en otra investigación, pues según indica Mitchell (2009), se trata de un modelo innovador en el campo. Además de esta acción legal, otra que ofrece buenos resultados en el corto plazo, consiste en desarrollar un contenido 
multiplataforma que dialogue con todas las plataformas digitales posibles. Para ello, el soporte digital es el comienzo más apropiado, aunque no es suficiente. A su vez, esto exige contratar a gente especialista en todo tipo de soportes y poner un énfasis especial en el registro de dónde y cuántas veces se reproducen los materiales de estas nuevas empresas.

Otra actividad clave en estas iniciativas de emprendimiento es la sindicación o redifusión de contenidos. Uno de los grandes temas pendientes de estas nuevas instituciones es conseguir una buena red de distribución de noticias, para incrementar su impacto en el espacio público. Algunas nonprofit como el Center for Public Integrity, The Investigative Reporting Workshop at American University, The Center for Investigative Reporting y ProPublica acordaron el 1 de julio de 2009 que su trabajo sería distribuido también por la agencia The Associated Press (Pérez-Peña, 2009). Con este tipo de acuerdo, mil 500 diarios de Estados Unidos podrían publicar estas historias potencialmente. Illinois Statehouse News (ISN) constituye otro caso de éxito, en asociación con medios tradicionales. Desde diciembre de 2009, su contenido diario ha sido utilizado por las estaciones de radio y de televisión en todo el estado, además de reutilizado por decenas de diarios (Stverak, 2010). Este tipo de acuerdos reforzaron su posicionamiento como una fuente confiable de información para su comunidad más próxima.

Aunque ninguno la declara como una actividad fundamental, la tutela de periodistas con experiencia es otro de los elementos importantes, por tres razones: la primera está relacionada con el tipo de información que se publica. Como se recuerda, la información manejada por el periodismo de investigación suele ser delicada, no solo por la envergadura de los temas que se tratan, sino también porque el proceso de publicación de una información de denuncia es un arte, puesto que, por lo general, está expuesta a grandes riesgos y a posibles denuncias penales o civiles. En ese sentido, contar con un profesional con experiencia, puede ayudar a ponderar las pruebas con las que se cuenta y a decidir si es el momento oportuno para publicar el tema. Además, no se trata de capturar la atención del público por un solo instante, sino también de retener la atención dosificando el material de la mejor manera. Y esta labor solo la puede llevar a cabo un experto. Finalmente, porque la pervivencia del periodismo de investigación depende de un capital que 
lo haga posible, pero también de periodistas que se sigan animando a apostar por él. Por lo investigado, en general las nonprofit están conformadas por equipos de tres a ocho profesionales, siempre bajo la tutela de un periodista experimentado (NonprofitJournalism.org, 2010), con amplia trayectoria en denuncia política o social.

El caso de Marianne Lavelle es un ejemplo. La periodista, con 11 años de experiencia en temas medioambientales, renunció a la prestigiosa revista U.S. News and World Report y pasó a formar parte del equipo de periodistas del Center for Public Integrity, en Washington D.C. Ahí pudo administrar mejor su tiempo para abordar temas complejos sobre su especialidad. Uno de sus artículos más recientes le permitió coordinar a tres reporteros. Gracias a este apoyo, el logro del que se siente más orgullosa es haber tenido tiempo para elaborar un cuadro que realmente aportara un valor formativo e ilustrativo a sus lectores, tarea que en su anterior trabajo hubiera resultado imposible (Halpert, 2009).

Otro ejemplo es el veterano Jim Scarantino, ${ }^{6}$ quien publicó para el NewMexicoWatchDog.org, ${ }^{7}$ una historia titulada "Los distritos fantasma”. En ella, reveló cómo 6 mil 400 millones de dólares estadounidenses, provenientes del plan de estímulo económico de Obama, fueron

6 Jim Scarantino, director y cofundador del New Mexico Watchdog es abogado de profesión y ha trabajado como autónomo para defender a aquellos que se encontraban solos ante la presión comercial o gubernamental. Justo cuando escribía este artículo Jim Scarantino acababa de redactar su renuncia como director de New Mexico Watchdog. Según relata en su carta, se retira para descansar de los distintos ataques personales y presiones que su labor le ha generado (Scarantino, 2010).

7 New Mexico WatchDog forma parte de la red de periodistas de investigación Watchdog.org financiada por Franklin Center for Government \& Public Integrity (Franklin Center for Government \& Public Integrity, 2009), una organización 501(C)3 sin ánimo de lucro dedicada a promover el periodismo en los nuevos medios. Por su parte, New Mexico Watchdog está financiado por la Fundación Río Grande, una organización sin ánimo de lucro dedicada a informar a los ciudadanos de su comunidad sobre la importancia de sus libertades individuales, los límites de su gobierno y las oportunidades económicas (Newmexico.watchdog.org, 2009). 
repartidos entre 440 distritos que en realidad no existían. Originalmente el dinero fue concebido para salvar y/o crear 30 mil puestos de trabajo, lo que equivalía a unos 225 mil dólares para cada puesto. Sin embargo, el 16 de noviembre de 2009 Scarantino descubrió que la lista de gastos de este dinero público mostraba síntomas de malversación (Recovery. gov, 2009). El supuesto peculado no fue reconocido por la administración Obama, sino tan solo por su vicepresidente, Joe Bidem, quien aseguró, pidiendo disculpas, que se trataba de errores en las cifras (Reichbach, 2009; Scarantino, 2009).

Finalmente, fue un periodista de investigación con 36 años de experiencia, quien descubrió en Nebraska que los profesores de una universidad estaban usando tarjetas de crédito financiadas por los contribuyentes para comprar unos billetes de avión, en primera clase, con destino a China, por 11 mil dólares estadounidenses (Stverak, 2010). El periodista Joe Jordan8 (Nebraska WatchDog, 2009) publicó el 29 de octubre de 2009 los gastos inapropiados descubiertos por el auditor Mike Foley. Según los informes del auditor, había pasajes comprados en primera clase con destino a China, Alemania y Hawaii. Esto incluso incumplía expresamente las normas de la universidad: 20 billetes de más de 5 mil dólares, cinco de más de 10 mil y uno de más de 15 mil; la suma de estos gastos indebidos ascendió a 173 mil dólares (Jordan, 2009).

Otro recurso imprescindible para la investigación que ha conseguido el periodismo sin ánimo de lucro es haber blindado el tiempo que los periodistas dedican a la investigación, independizándolo de las entregas secuenciales a las que está sometido el periodismo tradicional. Esto no solo sirve para investigar mejor sino también para confirmar las informaciones y para realizar una edición más minuciosa del material (Fest, 2009; Halpert, 2009; Mitchell, 2009; Stverak, 2010). Para nadie es un secreto que las organizaciones periodísticas tradicionales se han burocratizado y han desarrollado una compleja y tortuosa operatividad

8 Joe Jordan es un veterano director y periodista de investigación, con 36 años de experiencia en temas políticos. Ha recibido numerosos premios, entre los que se cuentan el Premio Walter Cronkite de periodismo político en televisión y el Premio Dupont de la Universidad de Columbia para la mejor investigación periodística (Nebraska WatchDog, 2009). 
diaria para la asignación de recursos (Mitchell, 2009). No sin enfrentamientos, los periodistas de investigación que trabajan en empresas tradicionales deben justificar por anticipado una serie de gastos que en un principio se pueden estimar, pero que luego, en función de cuánto se prolongue la investigación, se pueden contraer o triplicar.

Pasemos a hablar de los socios estratégicos. El principal aliado de este tipo de periodismo es el público. Los que apoyan el periodismo de investigación sin ánimo de lucro poseen un interés mayor por el funcionamiento de su comunidad (Mayer, 2010). Esto hace que contribuyan no solo con datos para las nuevas historias sino también compartiendo y recomendando la lectura del material publicado (Duckett, 2012). Se trataba de personas más comprometidas. Entre este público interesado, también se puede contar con especialistas por temas para la cocreación de contenidos, en interacción con los periodistas. Además de ellos, están las agencias de noticias, directamente relacionadas con la actividad de sindicación de contenidos. Si uno de los requisitos para el éxito de estas nuevas empresas es su impacto social, entonces tener buenas relaciones con agencias de noticias u otro tipo de organismos de difusión de información garantiza potencialmente su visibilidad. Esto por el lado de la difusión.

Por el lado de la investigación periodística, es deseable que este tipo de iniciativas se nutra de unas buenas relaciones que le permitan acceder a archivos desclasificados de los que echar mano en momentos de sequía informativa. Un ejemplo de ello es Wikileaks, cuyos importantes documentos revelados han sido el punto de partida perfecto para investigaciones de mayor calado social.

\section{Oferta}

También llamada "propuesta de valor". Como asegura Richard Tofel, al principio, el cometido principal de estas iniciativas es desarrollar investigaciones con mucha relevancia social, de alto impacto público (Fest, 2009). Es imprescindible que las investigaciones aborden estos temas y no otros, porque es precisamente aquí donde los medios tradicionales no llegan (Stverak, 2010). Junto a ello, también existe una imperiosa necesidad de conseguir publicar información más didáctica y cercana a la gente, reutilizable o republicable por gente común. Si algo caracteriza al periodista de investigación es que tiene un conocimiento muy profundo de los 
temas que trata y esto puede nublar su percepción didáctica del tema. Finalmente, el periodismo de investigación sin ánimo de lucro está llamado a realizar un seguimiento de aquellos temas que investiga, completando la documentación, o manteniendo la alerta social sobre el asunto.

\section{Clientes}

Hasta el momento, este tipo de periodismo ha conseguido identificar cinco tipos de público objetivo: a) agencias de noticias; b) medios tradicionales y regionales; c) capitales que promueven un trato más equitativo en la sociedad o simplemente fundaciones interesadas en la exoneración de impuestos; d) líderes de opinión; y finalmente, e) un público en general interesado en cuestiones de interés público.

Para atender a las demandas de la mayoría de ellos, las nonprofit han trabajado en tres canales de distribución distintos: a) los servicios de información y actualización multicanal, donde se contemplan desde boletines informativos que se distribuyen por correo electrónico, hasta aplicaciones para Ipad o smartphones, pasando por lectores $R S S$, etc.; b) los eventos, inicialmente concebidos para recaudar dinero de gente preocupada por su comunidad. Estos eventos han ido transformando al periodismo sin ánimo de lucro en una institución educativa, comprometida con la formación de la ciudadanía en valores fundamentales, y c) diseño de plataformas exclusivas para acceder a información en bruto de instituciones.

Con todo esto, ¿cómo han planteado las nonprofit su relación con el cliente? Hasta el momento, siguiendo el modelo Canvas, se han desarrollado dos vías de contacto: el primero es el autoservicio, es el propio cliente quien, haciendo uso de un programa informático, selecciona la información que quiere leer, la periodicidad con la que quiere hacerlo y la plataforma en la que va a hacerlo. Es el caso de The Texas Tribune, donde esta vía les ha generado más de 61 millones de páginas vistas desde su lanzamiento en 2009 (Duckett, 2012). En otros casos, estos nuevos medios conceden a los ciudadanos acceder libremente a las bases de datos de sus principales denuncias, para que realicen, por su cuenta, una vigilancia comunitaria (Duckett, 2012). Incluimos también en este apartado los boletines informativos vía correo electrónico, a los que se pueden suscribir los usuarios. Muchas veces estos boletines no se refieren a todo el contenido 
de la web de las nonprofit, sino solo al tema que preocupa a cada cliente y, como mucho, a una serie de recomendaciones relacionadas, siguiendo el estilo Amazon. El segundo es la cocreación. Aquí el usuario final introduce un input indispensable para dar por terminado el producto o servicio que se brinda y pasa a formar parte de la cadena de montaje. Lo más relacionado que logramos identificar en las iniciativas de periodismo de investigación sin ánimo de lucro es la creación de comunidades. Scott Lewis, director general de Voices of San Diego, indicó que las empresas informativas deben empezar a verse a sí mismas como educadoras, como "facilitadoras de la conversación", que promueven "un alto sentido del aprendizaje y el compromiso con los asuntos públicos. Nuestro nombre y nuestros objetivos están basados en nuestras investigaciones". Sin embargo, todavía batallan para resintonizarse con la comunidad y para conseguir que las personas dialoguen con ellos (McLellan, 2010b). Precisamente el diálogo que una comunidad puede desarrollar vía página web o redes sociales son una excelente moneda de cambio para estas empresas porque, entre otras cosas, incrementa la visibilidad de sus investigaciones. Una comunidad comprometida paga más y mejor, pero tal vez sea lo más difícil de conseguir en estos tiempos. Un estudio elaborado por el Pew Research Center (Stverak, 2010) indica que los estadounidenses mantienen su interés por las noticias, pero no están dispuestos a pagar por ellas, porque están seguros de poder obtenerlas gratis. Este es un concepto que debería empezar a cambiar.

\section{Financiamiento}

Una buena estructura financiera contempla no solo los ingresos sino también los gastos. La mayor parte de la bibliografía de referencia sobre este tema le exige al periodismo de investigación sin ánimo de lucro una mayor diversificación de sus fuentes de ingresos (McLellan, 2010b), precisamente porque la mayor parte de iniciativas se han intentado desarrollar sin haber conseguido un modelo plural de financiamiento (Osterwalder \& Pigneur, 2010). No obstante, analicemos primero la estructura de costes: cualquier negocio que acaba de empezar necesita dimensionar los egresos. A priori, sabemos que el periodismo de investigación es caro (Lewis, 2008). En unas empresas donde ya no se debe gastar en grandes equipos de retransmisión ni en grandes rotativas ni equipos de alta tecnología, 
el mayor gasto se despliega en dos aspectos: el acceso a bases de datos públicas o privadas, y el fichaje de personal cualificado y disponible. No olvidemos que se trata de un producto informativo de lujo, una especie de Rolex de la información periodística.

En cuanto a los ingresos, la principal fuente de financiamiento de la mayoría de nonprofit es el capital proveniente de fundaciones sin ánimo de lucro. Lo ideal, según los especialistas, es diversificar ese capital. Sin embargo, un extremo puede ser perjudicial a la hora de tomar decisiones: imagínese un acuerdo con 86 fuentes de financiamiento diferentes. Este es el número de socios con los que debe lidiar anualmente el Center for Public Integrity (CPI) (Walton, 2010). De todas formas, esto es lo menos común. El caso más frecuente es que al menos tres cuartas partes del presupuesto provengan de una sola fuente. ProPublica, financiada por un ex banquero Herbert Sandler y su mujer, Marion, es un claro ejemplo. "Ese es un modelo con el que solo podemos soñar", afirma Bergantino del New England Center (Walton, 2010).

Junto al capital de las fundaciones, algunas nonprofit ya trabajan para conseguir cuotas individuales de apoyo. Scott Lewis, de Voices of San Diego asegura que su presupuesto se estructura de la siguiente manera: 8\% de aportaciones individuales de usuarios o cuotas de membresía; $9 \%$ de publicidad; $62 \%$ de fundaciones; $17 \%$ de redes temáticas; 4\% de sindicación (NonprofitJournalism.org, 2010). A primera vista no parece una alternativa que, en este momento, se sostenga solo de las cuotas individuales. Sin embargo, si echamos un ojo a su tasa de renovación, alrededor de $70 \%$, según el propio Lewis (McLellan, 2010b), podríamos decir que se trata de una fórmula que aspira a consolidarse.

La siguiente modalidad es el patrocinio. Para Susan Mernit, fundadora de Oakland Local, los patrocinios constituyen una fuente permanente de ingresos (McLellan, 2010a). Sin embargo, no hay que perder de vista que la misma Mernit reconoce que sus fuentes más fiables de ingresos son la publicidad y los cursos (NonprofitJournalism.org, 2010). Paul Bass dijo que el New Haven Independent vende patrocinio, no avisos, lo que constituye $15 \%$ de su presupuesto (NonprofitJournalism.org, 2010). Además, conseguir patrocinios es también una oportunidad para asociarse con fuentes afines y para utilizar mejor su contenido periodís- 
tico. El extremo es atomizar el patrocinio. Este es el caso del periodista de investigación Joe Bergantino, director de WBZ-TV con sede en la Universidad de Boston, quien ha establecido "investigaciones periodísticas a pedido" de sus principales patrocinadores, con el objeto de capitalizar mejor sus labores cotidianas (Walton, 2010).

En el Centro de Periodismo de Investigación (Center for Investigative Reporting, CIR), Robert Rosenthal, su director ejecutivo, es una mezcla de director, dueño y vendedor. Él afirma que emplea la mitad de su tiempo consiguiendo fondos y la otra mitad mejorando historias y vendiéndolas a los distintos medios de comunicación tradicionales (Walton, 2010). A diferencia de otros medios sin ánimo de lucro, CIR ha concebido su material con ánimo de lucro.

Como decíamos antes, otra de las fuentes con las que se financia una nonprofit es la organización de eventos. Así lo considera, por ejemplo, John Thornton, director general del Texas Tribune (McLellan, 2010b). Su primer Texas Tribune Festival duró dos días y recaudó más de medio millón de dólares netos de taquilla, al vender cada entrada a 125 dólares (Duckett, 2012). El enfoque de esta institución es distinto porque se concibe como una empresa para formar a los ciudadanos (NonprofitJournalism. org, 2010; Phelps, 2011).

Además, las nonprofit se pueden financiar a través de tarifas de intercambio o licencia. Desde un principio, California Watch apostó por un modelo lucrativo de periodismo. Con este objetivo, vende a otras publicaciones los derechos de las historias que elaboran, a unas tasas realmente competitivas, que oscilan entre los 50 y los 500 dólares estadounidenses dependiendo de la envergadura de la historia y del comprador (McLellan, 2010a). De hecho, California Watch es de las pocas instituciones con una red de distribución bastante diversa y casi podríamos decir que a demanda de las necesidades de sus empresas (NonprofitJournalism.org, 2010). Evan Smith, director general de The Texas Tribune, opina también que el mayor valor del trabajo realizado por una nonprofit está en la republicación de su trabajo. "El valor real de lo que hacemos está en la segunda o tercera publicación de lo que hacemos como proveedores de distintas redes. Este es el valor real. Y el asunto más importante" (McLellan, 2010a). Ningún material periodístico tiene asegurado el éxito y solo se sabe de su impacto en el momento 
de su publicación. Es ahí cuando el número de solicitudes de reproducción del material determina su grado de repercusión. Desde sus inicios, en julio de 2008, ProPublica ha producido 17 grandes investigaciones, con múltiples repercusiones en distintos medios de tipo tradicional o electrónico. Los periodistas reciben dinero adicional en función de este indicador (Walton, 2010).

Por último, la publicidad constituye un viejo modelo de financiamiento para este tipo de empresas. Tradicionalmente, este modelo ha utilizado siempre al periodismo para llegar a distintos tipos de público de un modo organizado. No obstante, existe un riesgo al utilizar un viejo modelo de financiamiento: es probable que el periodismo no cambie lo suficiente como para sobrevivir. De hecho, como denuncia McLellan (2010b), tan pronto como estos nuevos medios digitales empiezan a facturar sumas importantes de publicidad, la preocupación por sintonizar con la audiencia pasa a un segundo plano.

Quedé conmocionada de cuán parecidos somos con los que consideramos como los medios tradicionales: usamos la palabra servicio a la comunidad; pero cuando el dinero de la publicidad fluye, difícilmente dependemos de nuestras comunidades y, como consecuencia, difícilmente nos hacemos eco de sus reales necesidades (McLellan, 2010a).

Como se puede apreciar, más que una nueva manera de hacer periodismo de investigación, el periodismo sin ánimo de lucro es una tendencia que busca innovar las formas de financiarlo. El reto mayor es el de diseñar modelos que no restrinjan su libertad para elegir temas y para publicarlos, libertad inherente al buen ejercicio del periodismo de investigación. Por lo pronto, como hemos constatado, ya existen nuevos intentos. Sin embargo, aún no podemos hablar de uno que funcione para todos los casos, sino que todo dependerá del tipo de producto $\mathrm{y}$ del concepto de beneficio que tengan sus fundadores.

\section{CONCLUSIONES}

1. El modelo Canvas es eficiente para descubrir los aciertos del modelo de periodismo sin ánimo de lucro. Existe un prejuicio generalizado 
de que este tipo de periodismo no ha diversificado sus fuentes de ingresos, aunque esto no es así. Las siete formas identificadas con el modelo Canvas hablan de un intento constante y paciente de parte de los profesionales para no depender solo del capital de las fundaciones, pese a que la mayor parte de los intentos todavía no hayan tenido el rendimiento esperado o al menos suficiente como para garantizar la subsistencia.

2. Hasta el momento, el modelo de organización de eventos parece ser el más acertado. Esto parte de una concepción distinta del periodismo, no solo como informador de la colectividad, sino también como capacitador en temas de interés público y como dinamizador de la participación. Además de beneficios económicos, los eventos proporcionan una masa crítica relevante para perfeccionar el trabajo diario y para prolongar el trabajo de los periodistas en nuevos formatos. La sintonía con la audiencia se transforma en algo fundamental para la pervivencia del periodismo de investigación sin ánimo de lucro. Parece ser que de este factor depende la constancia de las contribuciones económicas. En un principio estas no llegan a cubrir más de $10 \%$ del presupuesto de estas instituciones, pero se confía en que esto cambie con el paso del tiempo.

3. El trabajo en equipo vuelve a estar bajo la tutela de gente con experiencia que pueda solventar los principales escollos de una investigación periodística. Tener el financiamiento asegurado incide en un clima distinto dentro de estos nuevos equipos de trabajo, favorece la creatividad y promueve las iniciativas.

4. La diversificación de fuentes de ingresos plantea también un problema ético que no se resuelve solo con la transparencia a la hora de identificarlas, sino que debe empezar por ahí. Los periodistas se tienen que empezar a acostumbrar a rendir cuentas si son requeridos por la audiencia sobre cómo han solventado los costes de su trabajo de investigación.

Por todas estas razones debemos mirar con optimismo el futuro del periodismo de investigación sin ánimo de lucro. Ya solo el hecho de que se plantee realizar el mismo trabajo con un enfoque distinto merece una oportunidad que no le podemos negar. 


\section{Bibliografía}

American Society of News Editors-ASNE. (2010). The state of the news media. An annual report on American journalism. Recuperado el 19 de octubre de 2010 de http://www.stateofthemedia.org/2010/ chartland.php?msg $=1 \& \mathrm{id}=1314 \& \mathrm{ct}=1$ ine $\& \mathrm{dir}=\&$ sort $=\& \mathrm{c} 1=1 \& \mathrm{c} 2=$ $1 \& \mathrm{c} 3=0 \& \mathrm{c} 4=0 \& \mathrm{c} 5=0 \& \mathrm{c} 6=0 \& \mathrm{c} 7=0 \& \mathrm{c} 8=0 \& \mathrm{c} 9=0 \& \mathrm{c} 10=0 \& \mathrm{~d} 3=0 \&$ $\mathrm{dd} 3=1 \#$

Armstrong, P. (2008, 18 de noviembre). The media is dying. The Blog Hemld [Blog]. Recuperado el 29 de marzo de 2012 de https://twitter. com/\#!/THEMEDIAISDYING

Benton, J. (2010, 12 de abril). ProPublica's expensive story and deserved Pulitzer. Nieman Journalism Lab. Recuperado el 19 de octubre de 2010 de http://www.niemanlab.org/2010/04/ProPublicas-expensive-story-and-deserved-pulitzer/

Campos, F. (2010). Los nuevos modelos de gestión de las empresas mediáticas. Estudios sobre el mensaje periodístico, 16, 13-30.

Cohn, D. (2009, 24 de marzo). The "professional" bio. DigiDave [Blog]. Recuperado el 19 de octubre de 2010 de http://blog.digidave.org/about

Duckett, J. (2012, 13 de enero). The Texas Tribune case study: "Community Engagement" as journalism strategy. TexasInsider. org Recuperado el 5 de marzo de 2012 de http://www.texasinsider. org/?p=57332

Fest, M. (2009, 13 de junio). Knight Foundation Initiative seeks new models for investigative reporting. Knight Foundation. Informed communities \& Engaged. Recuperado el 19 de octubre de 2010 de http://www.knightfoundation.org/news/press_room/knight_press_ releases/detail.dot?id=348319

Franklin Center for Government \& Public Integrity. (2009, enero). Home page. Recuperado en septiembre de 2010 de http://www. franklincenterhq.org/

Greer, J. (2004). Advertising on traditional media sites: Can the traditional business model be translated to the Web? The Social Science Journal, 41, 107-113.

Halpert, J. (2009, 15 de septiembre). Nonprofit journalism model: A future of enviromental reporting? The Yale forum on climate 
change \& the media. Recuperado el 19 de octubre de 2010 de http:// www.yaleclimatemediaforum.org/2009/09/nonprofit-journalismmodel/

Hernández, R. (2010, 10 de octubre). Storify's Burt Herman on the evolution of reporter to entrepreneur. Online Journalism Review. Recuperado el 19 de octubre de 2010 de http://www.ojr.org/ojr/people/ webjournalist/201010/1894/

Houston, B. (2009). The case of The Winsconsin Center for investigative journalism. En New and innovative ideas of funding investigative journalism. Nueva York, EE.UU.: The Journalism School Columbia University. Recuperado el 10 de octubre de 2010 de http://www. watchdogconference.com/

Jordan, J. (2009, 29 de octubre). NU's credit cards: Used for some things they weren't supposed to buy. NebraskaWatchdog.org. Recuperado el 19 de octubre de 2010 de http://nebraska.watchdog. org/1873/1873/

Kaplan, D. (2007). Global investigative journalism: Strategies for support. A report to the center for international media assistance. Washington, D. C., EE.UU.: Center for International Media Assistance/National Endowment for Democracy.

Kuldell, H. (2007, 15 de junio). AAN announces AltWeekly Awards winners. Association of Alternative Newsweeklies. Recuperado el 23 de septiembre de 2010 de http://posting.altweeklies.com/aan/ aan-announces-altweekly-awards-winners/Article?oid=187877

Lewis, C. (2008). Seeking new ways to nurture the capacity to report. Nieman Reports.

Lozano, O. (2010, 27 de agosto). Investigative journalism in decline in U.S. Borderzine. Reporting Across Fronteras. Recuperado el 19 de octubre de 2010 de http://borderzine.com/2010/08/investigativejournalism-in-decline-in-u-s/

Mayer, J. (2010, 1 de octubre). The nonprofit world's "ladder of engagement". Reynolds Journalism Institute. Recuperado el 5 de enero de 2012 de http://rjionline.org/blog/nonprofit-worlds-ladder-engagement

McLellan, M. (2010a, 26 de abril). Seeking sustainability: The business of nonprofit journalism. News Leadership 3.0. Knight Digital 
Media Center [Blog]. Recuperado el 11 de septiembre de 2010 de http://www.knightdigitalmediacenter.org/leadership_blog/comments/20100426_seeking_sustainability_the_business_of_nonprofit journalism/

McLellan, M. (2010b, 23 de septiembre). Audience engagement and business sense are essential to success of local news start ups. News Leadership 3.0. Kight Digital Media Center [Blog]. Recuperado el 22 de octubre de 2010 de http://www.knightdigitalmediacenter.org/ leadership_blog/20100922_audience_engagement_and_business sense_are_essential_to_success_of/\#When:11:25:25Z

Mitchell, B. (2009, 2 de abril). Huffington post investigative team a nonprofit model in the making. Poynter online. Newspay. Recuperado el 19 de octubre de 2010 de http://www.poynter.org/column. asp? $\mathrm{id}=131$ \&aid $=161009$

Nebraska WatchDog. (2009, agosto). Nebraska WatchDog Staff. Recuperado el 19 de octubre de 2010, de Joe Jordan: http://nebraska. watchdog.org/about/staff/

Newmexico.watchdog.org. (2009, enero). About. Recuperado el 23 de septiembre de 2010 de http://newmexico.watchdog.org/about/

NonprofitJournalism.org. (2010, 26 de abril). Participant profiles. Profile Voices of San Diego. NonprofitJournalism.org. Recuperado el 19 de octubre de 2010 de http://nonprofitjournalism.org/2010/04/26/ profile-voices-of-san-diego.pdf

Osterwalder, A. \& Pigneur, Y. (2010). Business model generation. Hoboken, N. J., EE.UU.: Wiley \& Sons.

Pérez-Peña, R. (2009, 13 de junio). Nonprofit Journalism Bets Boost of The A.P. Media Decoder: Behind the Screens, Behind the Lines. NYT's Blogs [Blog]. Recuperado el 19 de octubre de 2010 http://mediadecoder.blogs.nytimes.com/2009/06/13/nonprofit-journalismgets-boost-de-ap/?scp=2\&sq=AP + ProPublica\&st=cse

Phelps, A. (2011, 25 de julio). For the Texas Tribune, "events are journalism" - and money makers. Nieman Journalism Lab. Recuperado el 5 de marzo de 2012 de http://www.niemanlab.org/2011/07/forthe-texas-tribune-events-are-journalism-and-money-makers/

Pressure on the presses. (2009, marzo). The Wall Street Journal. Recuperado el 19 de octubre 2010 de http://online.wsj.com/public/ resources/documents/NEWSPAPERS0903.html\#top 
ProPublica. (2008, junio). Steal our stories. ProPublica.org. Recuperado el 30 de marzo de 2012 de http://www.ProPublica.org/about/ steal-our-stories

Recovery.gov. (2009, 17 de febrero). Track the money. Recuperado el 22 de septiembre de 2010 de http://www.recovery.gov/Pages/home. aspx

Reichbach, M. (2009, 19 de noviembre). "Phantom districts" the result of "clerical errors." Biden says. The New Mexico Independent. Recuperado el 19 de octubre de 2010 de http://newmexicoindependent. com/41974/phantom-districts-the-result-of-clerical-errors-bidensays

Requejo, J. L. (2009). ¿Cómo se financia el periodismo de investigación en los Estados Unidos? Revista de Comunicación (VIII), 167181. Recuperado el 19 de octubre de 2010 de http://udep.edu.pe/ comunicacion/rcom/es/articulos/2009/Art167-181.html

Scarantino, J. (2009, 17 de noviembre). $\$ 6.4$ billions stimulus goes to phantom districts. Watchdog.org. Recuperado el 23 de septiembre de $2010 \mathrm{de} \mathrm{http://watchdog.org/1530/6-4-billion-stimulus-goes-to-}$ phantom-districts/

Scarantino, J. (2010, 22 de septiembre). Jim Scarantino Departs New Mexico Watchdog. New Mexico Watchdog. Recuperado el 23 de septiembre de 2010 de http://newmexico.watchdog.org/3892/jimscarantino-departs-new-mexico-watchdog/

Shepherd, T. (2010, 24 de febrero). Department of Homeland Security loses over 1,000 computers in one year. Big Government. Recuperado el 10 de octubre de 2010 de www.breitbart.com/BigGovernment/2010/02/24/Dept-of-Homeland-Security-Loses-over1-000-Computers-in-One-Year

Shepherd, T. (2010, 24 de febrero). Homeland Security loses over 1,000 computers in one fiscal year. Independence Institute. Recuperado el 19 de octubre de 2010 de http://investigates.i2i.org/2010/06/25/ homeland-security-loses-over-1000-computers-in-one-fiscal-year/

Stverak, J. (2010, 13 de abril). Investigations and explanations-two journalism tasks where nonprofits can thrive. Online journalism review. Recuperado el 11 de septiembre de 2010 de http://www.ojr. org/ojr/people/stverak/201004/1841/ 
Walton, M. (2010, septiembre). The Nonprofit Explosion. American Journalism Review. Recuperado el 27 de septiembre de 2010 de http://www.ajr.org/Article.asp?id=4906

Wasserman, E. (2011, 11 de abril). Is nonprofit investigative journalism sustainable? Nonprofit Quarterly. Recuperado el 11 de septiembre de http://www.nonprofitquartley.org/updates/11316-is-nonprofit-investigative-journalism-sustainable.html

Fecha de recepción: 25/05/11. Aceptación: 01/10/11. 